\title{
Shape of threadlike molecules in solution, and relationship between solution viscosity and molecular weight
}

\author{
Ichiro Sakurada* \\ Polymer Journal (2012) 44, 5-10; doi:10.1038/pj.2011.121
}

Translated reproduction of Sakurada, I. Empirical Relationship between Intrinsic Viscosity and Molecular Weight [Youeki nendo to bunshiryo no kankei]. In: Proceedings of the 5th Meeting** of Kyoto Imperial University, Research Institute for Chemical Fibers, Japan. [Nihon Kagaku Sen-i Kenkyusho], 33-44, 1940, Kyoto

Translation by Yutaka Sakurada

In recent years, the results of scientific research have indicated that the substances that form natural fibers, and regenerated man-made fibers, are chemically composed of long molecules, which are referred to as threadlike molecules. Synthetic threadlike molecules from which synthetic fibers are prepared cannot be derived directly from natural materials but are synthesized by chemically bonding many small molecules, that is, by polymerization reactions.

The difficulties of the preparation of yarn by spinning fibers mainly depend on factors such as the thickness, length and crimping of a single fiber. Similarly, when fibers are spun from a spinning solution, the important factors are the thickness, length and shape of the threadlike molecules. The length and thickness of a threadlike molecule can be estimated from the molecular weight and specific gravity, based on its chemical structure. The shape of the molecule, however, cannot currently be determined with high precision. Nevertheless, the solution viscosity and the diffusion constant provide useful specific information about the shape. Today, I would mainly like to report the results of my recent considerations of the relationship between solution viscosity and molecular shape, based on the experimental results from H. Staudinger's school.

\section{RELATIONSHIP BETWEEN SOLUTION VISCOSITY AND MOLECULAR WEIGHT}

Various relationships between molecular weight (or molecular length) and solution viscosity, which depends on the molecular shape and other factors, have been found both theoretically and experimentally. These relationships are collectively shown in Table 1.

Table 1 shows the relationships between the solution viscosity $\left(\eta_{\mathrm{sp}} / \mathcal{c}\right)$ and the degree of polymerization $(P)$ of the solute. The degree of polymerization is used instead of the molecular weight or the molecular length for the following reason: in a homologous series of polymers, the thickness of the threadlike molecule is constant, regardless of the degree of polymerization, and the straight elongated length of the threadlike molecule is proportional to the molecular weight or the degree of polymerization.

The formula in column no. 1 of Table 1 , subsequently abbreviated to 'formula (1)', describes the relationship between the viscosity and the length of the rod or thread when the solute threadlike molecule is not bent. Unlike formula (1), formula (2), which is known as Staudinger's viscosity law, is experimentally determined. Staudinger assumed (without sufficient theoretical basis) that formula (2) holds for straight threadlike molecules, according to a very simple idea that the more complicated bent form would never give such a simple formula. In contrast with formula (2), however, the derivation of formula (1) was purely theoretical and indicates that the viscosity $\left(\eta_{\mathrm{sp}} / c\right)$ for the straight form of the molecules is not proportional to the degree of polymerization $(P)$ but to the square of $P$. From these two results, H Mark and W Kuhn asserted that the molecule do not dissolve in the straight form assumed by Staudinger, but it must be considerably bent. Sakurada further clarified theoretically that the threadlike molecule bends and shrinks in proportion to the cube root of the degree of polymerization, indicating that it agrees formally with the experimental formula of $\mathrm{H}$ Staudinger (I Sakurada, Z Physik. Chem., B38, 407-423, 1938). [Editorial note: The original reference 'I Sakurada and M Taniguchi, Z. Physik. Chem. A. 1937, 179, 227' seems incorrect because the title is 'Über die Diffusion von heterodispersen Stoff (On the Diffusion of Heterodispersion Matter)'. The correct paper cited above is 'Über die Viscosität der Lösungen von Fadenmolekülen (On the Solution Viscosity of Threadlike Molecules)'.] 
Table 1 Relationships between viscosity of solution and degree of polymerization of molecules (various viscosity formulae)

\begin{tabular}{|c|c|c|c|c|}
\hline Nos. & 1 & 2 & 3 & 4 \\
\hline Particle shape & Straight rod shape & Considerably bent threadlike molecule & Entirely freely bendable threadlike molecule & Dense spherical particles \\
\hline Method inducing formula & Purely theoretical & Empirical & Purely theoretical & Purely theoretical \\
\hline Person(s) inducing formula & Eisenschitz, Kuhn, Guth & Staudinger & Kuhn & Einstein \\
\hline Formula & $\eta_{\mathrm{sp}} / c=K P^{2}$ & $\eta_{\mathrm{sp}} / c=K P$ & $\eta_{\mathrm{sp}} / c=K P^{1 / 2}$ & $\eta_{\mathrm{sp}} / c=K P^{0}=K$ \\
\hline Applicable examples & None & $\begin{array}{l}\text { Cellulose and its derivatives, } \\
\text { polystyrene and lower chain molecules }\end{array}$ & Unknown & $\begin{array}{l}\text { Various low-molecular } \\
\text { substances, glycogen }\end{array}$ \\
\hline
\end{tabular}

Formula (3) was derived purely theoretically by W Kuhn under the assumption that the threadlike molecule may be entirely freely bent as a result of the hypothetical free rotation of each bond. Although no examples applicable to this formula have been reported to date, I will primarily discuss this issue here.

Formula (4) is a famous viscosity formula by Einstein, which requires that viscosity is entirely independent of the degree of polymerization. If the threadlike molecules are freely bent into dense spherical particles, the solution volume occupied by the substance per unit weight does not depend on the degree of polymerization. In such a case, formula (4) may be applicable. It is known that for glycogen, which is a polymer material, formula (4) holds true. Staudinger's group interpreted this agreement as follows: formula (4) does not hold good because of bending of the molecule, but rather because the molecules are highly branched, yielding considerably dense spheres. The author also considers that such an interpretation is reasonable.

When Table 1 is considered as a whole, it may be observed that the relationship between the viscosity and the degree of polymerization of a molecule can be generally expressed by the relationship

$$
\eta_{\mathrm{sp}} / c=K P^{n}
$$

where $n$ is in the range from 0 to 2 . Thus, it is assumed that as $n$ approaches 0 from 2, the molecules become more and more bent. Furthermore, it should be noted that in the formulae cited thus far, the value $\eta_{\mathrm{sp}} / c$ ideally means $\left(\eta_{\mathrm{sp}} / c\right)_{c \rightarrow 0}$.

\section{VARIOUS VINYL AND ACRYLIC SYNTHETIC THREADLIKE MOLECULES}

The synthetic threadlike polymer that is most frequently used to validate the experimental formula of Staudinger is polyvinylbenzene or polystyrene. Thus, it has been confirmed by many examples using this polymer that formula (2) holds well, though the value of the constant $K$ changes somewhat, depending on the polymerization condition or the polymerization temperature, which causes slight variation of the degree of branching. In every example, including polystyrene and the following polymers, the degree of polymerization is determined by osmometry.

Recently, H Staudinger has observed that many synthetic threadlike molecules of vinyls and acrylics (as shown below) do not follow the conventional viscosity law (H Staudinger, H Warth, J. Prakt. Chem., 155, 261 (1940)):

- Polyvinylchloride

- Post-chlorinated polyvinylchloride (PeCe fiber material)

- Polyvinylchloride acetate (Vinyon material)

- Polyvinylacetate

- Polyvinylalcohol ('Go-sei Ichigo (Synthetic No. 1)’ fiber material)

- Polymethylacrylate

- Polymethylmethacrylate

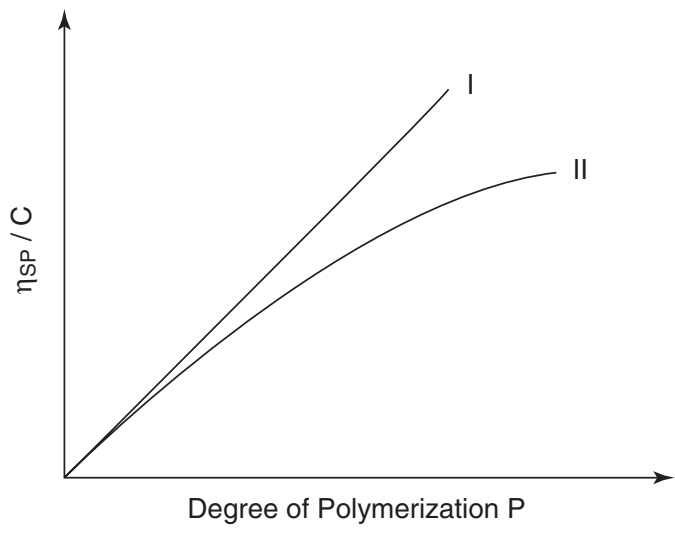

Figure 1 Relationship between viscosity $\eta_{\mathrm{sp}} / c$ and the degree of polymerization $P$. Curve I represents a straight line, in agreement with the viscosity law of Staudinger, and curve II shows an example deviating from the law.

If a compound conforms to the viscosity law of Staudinger, a straight line passing through the origin will be observed in the graph of the viscosity $\left(\eta_{\mathrm{sp}} / c\right)$ as a function of the degree of polymerization $P$, such as curve I in Figure 1. For any of the abovementioned synthetic, threadlike molecules, however, the relationship becomes similar to that seen in curve II, in which the viscosity is not proportional to the degree of polymerization (Figure 1); rather, it levels off in the region of high degrees of polymerization. Staudinger and his colleagues have raised the following two possible explanations:

(1) The threadlike molecule is considerably bent.

(2) The threadlike molecule is considerably branched.

Despite their various studies, they have not yet reached a clear conclusion.

We will further consider this problem in greater detail by utilizing formula (5). Taking the logarithm of both sides of formula (5), the following equation is obtained:

$$
\log \left(\eta_{\mathrm{sp}} / c\right)=\log K+n \log P
$$

From this equation, $n$ can be determined by drawing the $\log \left(\eta_{\mathrm{sp}} / c\right)$ vs $\log P$ curve, so that the $n$ values can be used to make specific considerations below.

\section{Polyvinylchloride}

When the relationship between $\log \left(\eta_{\mathrm{sp}} / c\right)$ and $\log P$ is plotted for a solution of fractionated polyvinylchloride in tetrahydrofuran, the relationship never displays one straight line, as shown in Figure 2. 
However, if data are carefully observed (Figure 2), the entire trend can be represented well by two straight lines, as indicated in the Figure. When the values of $n$ are determined from the inclination of these straight lines, the following interesting results are obtained:

In the region of $P$ less than $500, n=0.97 \cong 1$.

In the region of $P$ more than $500, n=0.45 \cong 0.50$.

These results may be interpreted by considering Table 1 and formula (5) as follows: In the region in which the degree of polymerization is less than 500 , the threadlike molecules are slightly bent, in accordance with the experimental formula of Staudinger

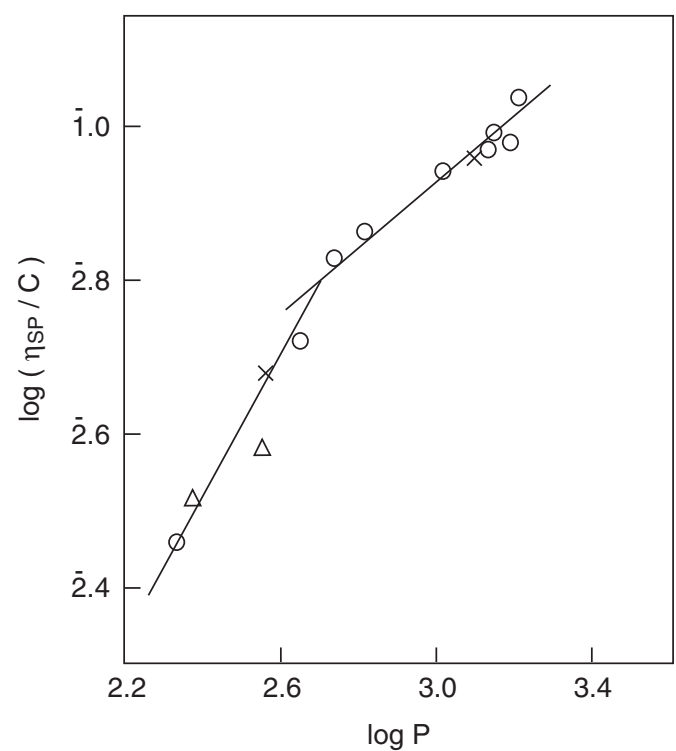

Figure 2 Viscosity $\eta_{\mathrm{sp}} / c$ vs degree of polymerization $P$. Tetrahydrofuran solution of fractionated polyvinylchloride. (Editorial note: The figures on the ordinate are represented in a peculiar way. The bar on a cardinal number indicates that only the bar-capped integer is minus, but a decimal part without bar is positive. Hence, for example, $\overline{2} .4$ means -1.6 . Numerical values are similarly represented in the following figures.) (as in the case of polystyrene). In the region over 500, the molecules are significantly bent such that they are in an entirely free bending state, as shown in Kuhn's model. Thus, the experimental results mentioned above formally provide an actual example of Kuhn's purely theoretical formula for the freely bending threadlike molecules. A similar relationship is also found for non-fractionated polyvinylchloride. The values of $n$ and the other parameters are summarized in Table 2.

In the case of a solution of fractionated polyvinylchloride in dioxane, the entire trend can be expressed by two straight lines, as shown in Figure 3. The obtained $n$ values are as follows:

For the region of $P$ less than $300: n=1.06 \cong 1$.

For the region of $P$ more than $300: n=0.154$.

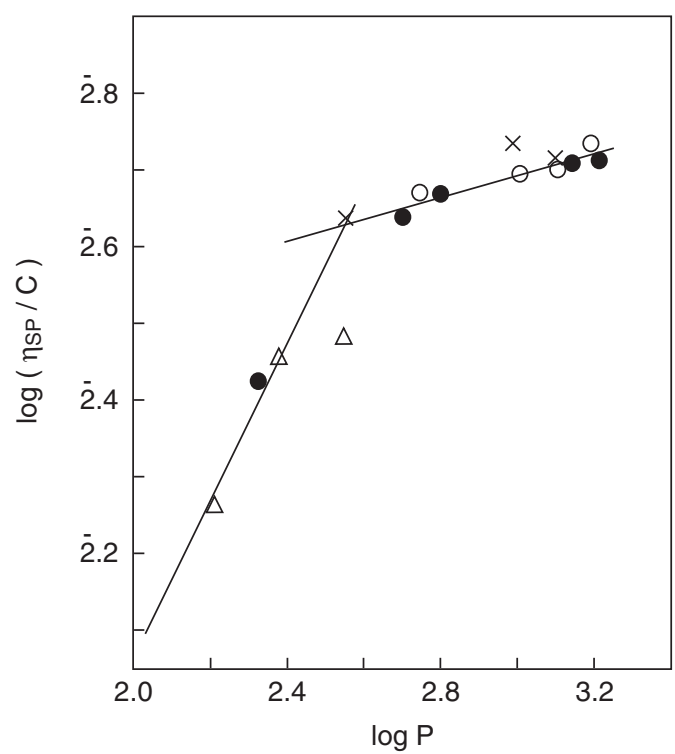

Figure 3 Viscosity $\eta_{\mathrm{sp}} / c$ as a function of the degree of polymerization $P$. Dioxane solution of fractionated polyvinylchloride.

Table 2 Exponent $n$ of the formula $\eta_{\mathrm{sp}} / c=K P^{n}$, indicating the relationship between viscosity and the degree of polymerization of synthetic, threadlike molecules in solution

\begin{tabular}{|c|c|c|c|c|}
\hline \multirow[b]{2}{*}{ Substance } & \multirow[b]{2}{*}{ Solvent } & \multicolumn{2}{|c|}{$n$} & \multirow{2}{*}{$\frac{n \text { (round) }}{\text { High degree of polymerization }}$} \\
\hline & & Low degree of polymerization & High degree of polymerization & \\
\hline Polyvinylchloride (raw) & Dioxane & 0.95 & 0.20 & - \\
\hline Polyvinylchloride (raw) & Tetrahydrofuran & 1.01 & 0.61 & $1 / 2$ \\
\hline Polyvinylchloride (fractionated) & Dioxane & 1.06 & 0.154 & - \\
\hline Polyvinylchloride (fractionated) & Tetrahydrofuran & 0.97 & 0.452 & $1 / 2$ \\
\hline Post-chlorinated polyvinylchloride & Dioxane & 0.86 & 0.298 & $1 / 3$ \\
\hline Post-chlorinated polyvinylchloride & Tetrahydrofuran & 0.90 & 0.379 & $1 / 3$ \\
\hline Polyvinylchloride acetate & Acetone & & 0.65 & $2 / 3$ \\
\hline Polyvinylacetate & Acetone & & 0.666 & $2 / 3$ \\
\hline Polyvinylalcohol & Water & & 0.70 & $2 / 3$ \\
\hline Polymethylacrylate & Acetone & & 0.714 & $2 / 3$ \\
\hline Polymethylmethacrylate & Chloroform & & 0.59 & $1 / 2$ \\
\hline Polymethylmethacrylate & Acetone & & 0.57 & $1 / 2$ \\
\hline Polystyrene & Benzene & 1 & 1 & 1 \\
\hline Polystyrene & Carbon tetrachloride & 1 & 1 & 1 \\
\hline
\end{tabular}


Thus, when the degree of polymerization is low, $n$ is equal to 1 , as in the case of the tetrahydrofuran solution. However, for higher degrees of polymerization, $n$ becomes significantly smaller than $1 / 2$. The decrease of $n$ may be interpreted as a result of the molecules approach toward the state of dense spherical particles because of the intramolecular association caused by their free bending. In fact, dioxane is a nearly nonpolar liquid that has little ability to solvate polyvinylchloride. There is significant evidence to support the occurrence of intramolecular association in this case, which will be discussed in detail at another time. The results for the non-fractionated substances are nearly the same.

Post-chlorinated polyvinylchloride and polyvinylchloride acetate It was also possible to express the entire trend for post-chlorinated polyvinylchloride, which is the raw material of Pe-Ce fibers, with two straight lines, though the graph is not shown. In this case, the number of observed points was not as high as in the case of polyvinylchloride; as a result, the reliability of the estimated $n$ values is somewhat lower. Nevertheless, they are obtained as follows:

In the range of lower degrees of polymerization:

$n=0.90 \cong 1$ in tetrahydrofuran.

$n=0.86 \cong 1$ in dioxane.

In the range of higher degrees of polymerization:

$n=0.379$ in tetrahydrofuran.

$n=0.298$ in dioxane.

The lower range of the degree of polymerization gives $n=1$, is in agreement with Staudinger's experimental formula, whereas in the higher range, $n \cong 1 / 3$, in both tetrahydrofuran and dioxane. The latter value is still smaller than the value for free bending given by Kuhn's theory, but it is higher than the value for the dioxane solution of polyvinylchloride. Although an immediate discussion of the causes of this phenomenon may be somewhat unreasonable, it does not seem difficult to qualitatively understand the results described above. It may be considered that both the solvation and association forces of the threadlike molecules increased as a result of the post-chlorination process, though the solvation effect was more significant in tetrahydrofuran, whereas the effect of association was more pronounced in dioxane.

In polyvinylchloride acetate, the raw material for the production of vinyon, $n$ is nearly equal to $2 / 3$, though the observed data are still unconfirmed. This result is the same as that for polyvinylacetate and polyvinylalcohol, which will be discussed below. The effect is presumably caused by the increased solvation ability because of the

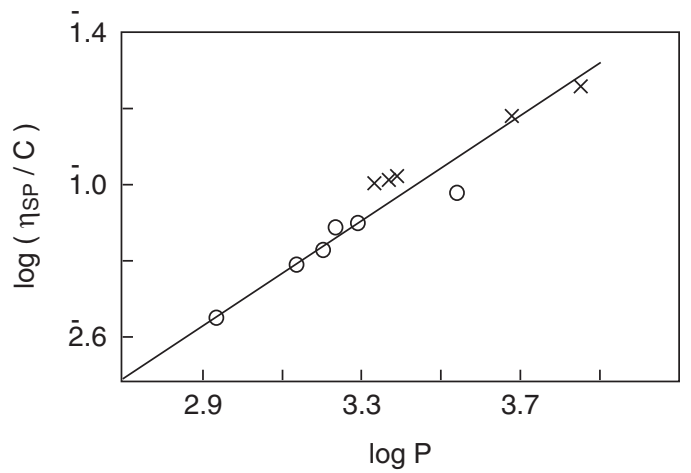

Figure 4 Viscosity $\eta_{\mathrm{sp}} / c$ as a function of the degree of polymerization $P$. Acetone solution of polyvinylacetate. copolymerized component, resulting in the suppression of the free bending.

\section{Polyvinylacetate and polyvinylalcohol}

The relationships between $\log \left(\eta_{\mathrm{sp}} / \mathcal{c}\right)$ and $\log P$ for polyvinylacetate and polyvinylalcohol are shown in Figures 4 and 5, respectively.

In both the cases, it is possible to express the entire trend by one line, according to formula (6). The values of $n$ for an acetone solution of polyvinylacetate and an aqueous solution of polyvinylalcohol are 0.666 and 0.70 , respectively, both of which are nearly equal to $2 / 3$. This result indicates that these polymers are more flexible than polystyrene, which follows Staudinger's viscosity law, but less flexible than polyvinylchloride. The former is easily understood from the fact that polystyrene has many benzene rings as side chains, whereas the latter may be attributed to a solvation effect. The polymer molecules of polyvinylacetate in acetone or polyvinylalcohol in water are strongly solvated. The solvating low molecules work as if they were side chains of the polymers themselves, which reduces the flexibility of the polymer chains. In addition, the intramolecular associations of these threadlike molecules are difficult to consider.

\section{Polymethylacrylate and polymethylmethacrylate}

The plots of $\log \left(\eta_{\mathrm{sp}} / c\right)$ vs $\log P$ for acetone solutions of polymethylacrylate and polymethylmethacrylate are shown in Figures 6 and 7, respectively, both of which consist of one straight line. Their $n$ values are 0.714 and 0.57 , respectively (Figures 6 and 7). Simple consideration suggests that the latter should be less flexible because one of the skeletal protons of the former is substituted with $\mathrm{CH}_{3}$. However, the $n$ value for the latter is somewhat smaller and nearly equal to $n=1 / 2$ for the free bending case. This difference in the values of $n$ may occur because the solvation effect is more significant in polymethylacrylate. If lower degrees of polymerization had been investigated in examples (c and $\mathrm{d}$ ), the $n=1$ regions would have been found.

\section{GENERAL FORMULA GIVING THE RELATIONSHIP BETWEEN VISCOSITY AND DEGREE OF POLYMERIZATION OF A MOLECULE}

As discussed above, the relationship between viscosity and the degree of polymerization of a molecule can be most clearly expressed by formula (5). In practice, however, the observed curves of $\log \left(\eta_{\mathrm{sp}} / c\right)$ vs $\log P$ do not display such ideal straight lines. This deviation may

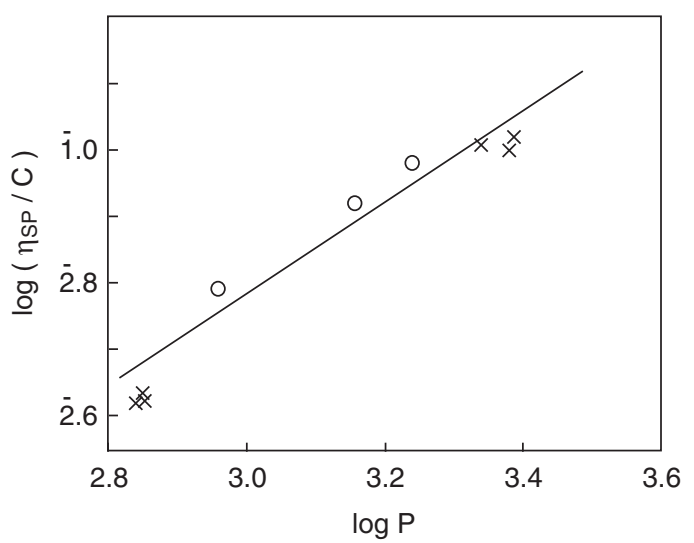

Figure 5 Viscosity $\eta_{\mathrm{sp}} / c$ as a function of the degree of polymerization $P$. Aqueous solution of polyvinylalcohol. 
mainly occur because of the molecular weight distribution (or polydispersity). The degrees of polymerization reported for the samples examined thus far used have been the average values, termed polymereinheitlich (of uniform or centralized degree of polymerization) by Staudinger, meaning that they are not completely uniform in molecular weight. Some branching of the threadlike molecules may also affect this deviation. Nevertheless, without considering the branching effect, we have been able to quantitatively explain the case in which the observed data contradict Staudinger's viscosity law in terms of the bending of threadlike molecules and their intramolecular associations. We have also been able to demonstrate that Kuhn's formula for free bending is applicable to some of the above examples, at least in the formal sense. The $n$ values found in the previous section

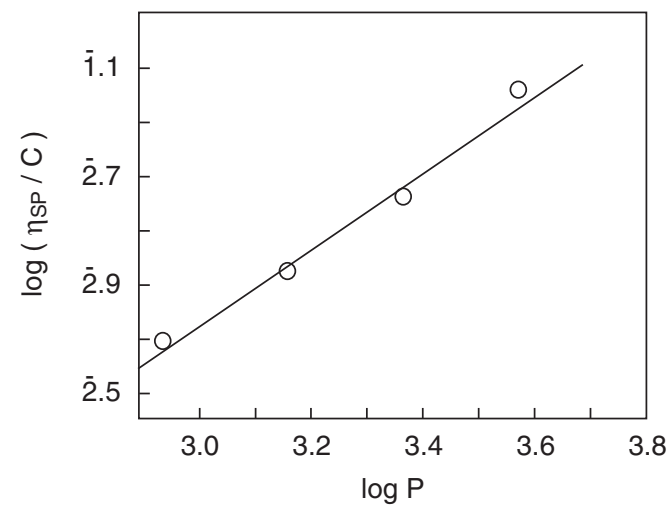

Figure 6 Viscosity $\eta_{\mathrm{sp}} / c$ as a function of the degree of polymerization $P$. Acetone solution of polymethylacrylate.

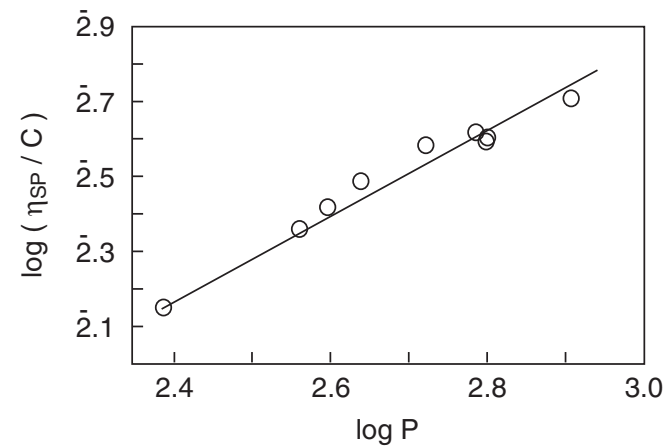

Figure 7 Viscosity $\eta_{\mathrm{sp}} / c$ as a function of the degree of polymerization $P$. Acetone solution of polymethylmethacrylate.

Table 3 Tetrahydrofuran solution of polyvinylchloride (raw products), $n=1 / 2$

\begin{tabular}{lrrrrrrrrr}
\hline$P$ & 2500 & 2200 & 1640 & 1550 & 1400 & 1270 & 980 & 640 & 600 \\
$\left(\eta_{\mathrm{sp}} / c\right) \times 10^{3}$ & 135 & 140 & 113 & 99 & 103 & 95 & 105 & 75 & 71 \\
$K \times 10^{4}$ & 260 & 298 & 279 & 241 & 275 & 266 & 332 & 293 & 290 \\
$P_{\text {calc }}$ & 2520 & 2720 & 1770 & 1250 & 1480 & 1250 & 1530 & 780 & 700 \\
$P$ & 560 & 450 & 365 & 350 & 380 & 230 & 260 & 245 & 220 \\
$\left(\eta_{\mathrm{sp}} / c\right) \times 10^{3}$ & 69 & 54 & 48 & 39 & 39 & 34 & 37 & 33 & 33 \\
$K \times 10^{4}$ & 292 & 250 & 252 & $(206)$ & $(206)$ & $(211)$ & $(230)$ & $(212)$ & $(216)$ \\
$P_{\text {calc }}$ & 658 & 400 & 320 & - & - & - & - & - & - \\
\hline
\end{tabular}

Mean value of $K=0.00283$ are collectively shown in Table 2 . In the last column, Table 2 the rounded values of ' $n$ (round)', such as $1 / 2,1 / 3$ and $2 / 3$, corresponding to the observed values, are given for the convenience of the following calculations. The use of such round numbers is reasonable because the

Table 4 Tetrahydrofuran solution of polyvinylchloride (fractionated products), $n=1 / 2$

P $\quad 15501340104056016401400640450 \quad 2201270365 \quad 360 \quad 246$ $\begin{array}{llllllllllllll}\left(\eta_{\mathrm{sp}} / c\right) \times & 98 & 97 & 91 & 69 & 113 & 103 & 75 & 54 & 29 & 95 & 48 & 39 & 33\end{array}$ $10^{3}$

$\begin{array}{lllllllllllll}K \times 10^{4} & 249 & 266 & 282 & 290 & 279 & 276 & 296 & 255 & (196) & 257 & 252 & \text { (205) (210) }\end{array}$ $P_{\text {calc }} 13301300115065817601470780405$ - 1250320 - _

Mean value of $K=0.00285$.

Table 5 Dioxane solution of post-chlorinated polyvinylchloride, $n=1 / 3$

\begin{tabular}{lcccccccc}
\hline$P$ & 3100 & 2800 & 1270 & 970 & 800 & 800 & 760 & 255 \\
$\left(\eta_{\mathrm{sp}} / c\right) \times 10^{3}$ & 119 & 110 & 100 & 86 & 77 & 62 & 84 & 32 \\
$K \times 10^{4}$ & 81.6 & 78.0 & 92.2 & 87.0 & 83.0 & 66.8 & 92.0 & $(50.4)$ \\
\hline
\end{tabular}

Table 6 Acetone solution of polyvinylchloride acetate, $n=2 / 3$

\begin{tabular}{lcccc}
\hline$P$ & 1700 & 1100 & 950 & 1150 \\
$\left(\eta_{\mathrm{sp}} / c\right) \times 10^{3}$ & 100 & 78 & 74 & 69 \\
$K \times 10^{4}$ & 7.04 & 7.32 & 7.68 & 6.23 \\
\hline
\end{tabular}

Table 7 Acetone solution of polyvinylacetate, $n=2 / 3$

P $\quad 74003650180021001450165087075002250500026002450$

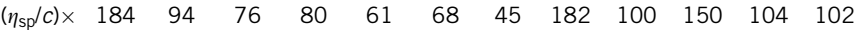
$10^{3}$

$\begin{array}{lllllllllllll}K \times 10^{4} & 4.84 & 3.97 & 5.14 & 4.87 & 4.78 & 4.86 & 4.95 & 4.75 & 5.81 & 5.14 & 5.50 & 5.60\end{array}$

Table 8 Aqueous solution of polyvinylalcohol, $n=2 / 3$

$\begin{array}{lccccccccc}P & 1750 & 1450 & 910 & 2200 & 2450 & 2400 & 700 & 700 & 700 \\ \left(\eta_{\mathrm{sp}} / c\right) \times 10^{3} & 95 & 84 & 63 & 104 & 105 & 102 & 43 & 42 & 42 \\ K \times 10^{4} & 6.53 & 6.51 & 6.64 & 6.16 & 5.78 & 5.68 & 5.38 & 5.23 & 5.30\end{array}$

Table 9 Acetone solution of polymethylacrylate, $n=2 / 3$

\begin{tabular}{lcccc}
\hline$P$ & 3750 & 2350 & 1450 & 870 \\
$\left(\eta_{\mathrm{sp}} / c\right) \times 10^{3}$ & 114 & 73 & 53 & 39 \\
$K \times 10^{4}$ & 4.71 & 4.13 & 4.14 & 4.29 \\
\hline
\end{tabular}

Table 10 Polymethylmethacrylate in chloroform and in acetone solution, $n=1 / 2$

\begin{tabular}{lccccccccc}
\hline$P$ & 2500 & 1580 & 1540 & 1440 & 1140 & 770 & 640 & 540 & 250 \\
$\begin{array}{l}\text { Chloroform } \\
\left(\eta_{\mathrm{sp}} / c\right) \times 10^{3}\end{array}$ & 126 & 87 & 90 & 91 & 83 & 69 & 56 & 50 & 27 \\
$K \times 10^{4}$ & 25.2 & 21.9 & 22.9 & 24.0 & 24.6 & 24.9 & 22.2 & 21.5 & $(17.1)$ \\
$\begin{array}{l}\text { Acetone } \\
\left(\eta_{\mathrm{sp}} / c\right) \times 10^{3}\end{array}$ & 51 & 40 & 39 & 41 & 39 & 31 & 26 & 23 & 14 \\
$K \times 10^{4}$ & 10.0 & 10.1 & 9.9 & 10.6 & 11.6 & 11.2 & 10.3 & 9.9 & $(8.9)$ \\
\hline
\end{tabular}


observed points are usually somewhat scattered, such that the obtained $n$ value slightly varies depending on how a line is drawn through the points. Thus, the observed $n$ value indicates only one of the possible values estimated by consideration of the entire trend. In the table, an example of polystyrene is also shown for the sake of comparison.

Furthermore, the constants $(K)$ calculated using the $n$ (round) values in Table 2 are shown in Tables 3-10. In every case it is confirmed that they are nearly constant (Tables 3-10), regardless of the degree of polymerization. Using the values of $n$ and $K$ thus obtained, the degrees of polymerization $\left(P_{\text {calc }}\right)$ were calculated only for polyvinylchloride. These values are also shown in Table 3 and found to all agree well with the experimental $P$ values.

Furthermore, we have examined the $K$ values when $n=1 / 2$. For the tetrahydrofuran solution of polyvinylchloride, the average value of $K$ is determined to be 0.00284 from Tables 3 and 4; for the chloroform solution of polymethylmethacrylate, the average value of $K$ becomes 0.00234 from Table 10. These numerical values are nearly equal, suggesting that $K$ is a characteristic constant. If we assume that, in polyvinyls or polyacryls, the seventh or eighth carbon of a polymer chain bends entirely freely against the first carbon (as indicated below),

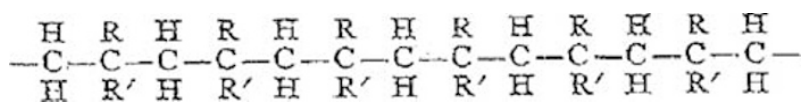

we would be able to derive the above constant purely theoretically. Such a derivation seems the most probable, even after other factors are considered. In this regard, we would like to further discuss this topic in detail elsewhere. 